\title{
The Capacity Region of a Product Gaussian Broadcast Channel with Degraded Message Sets
}

\author{
Jae Won Yoo and Tie Liu \\ Dept. of Electrical \& Computer Engineering \\ Texas A\&M University \\ College Station, TX 77843 \\ E-mail: \{yoojw78,tieliu\}@tamu.edu
}

\author{
Yingbin Liang \\ Dept. of Electrical Engineering \& Computer Science \\ Syracuse University \\ Syracuse, NY 13244 \\ E-mail: yliang06@syr.edu
}

\begin{abstract}
A product Gaussian broadcast channel with three receivers and degraded message sets is considered. A single-letter characterization of the capacity region was obtained in a recent work of Nair and El Gamal based on superposition coding and indirect decoding. Through Lagrangian theory and an extremal entropy inequality, the single-letter expression of Nair and EI Gamal is evaluated for the specific product Gaussian model, which leads to an explicit characterization of the capacity region.
\end{abstract}

\section{INTRODUCTION}

Broadcast is a fundamental nature of wireless communication: any receiver within the transmission range can listen to the source and potentially decode some of the messages. With appropriate coding architecture, the broadcast nature of wireless communication can be used to the advantage of simultaneously transmitting to several receivers at high rates. Understanding the limits and the appropriate coding architectures that can harness the broadcast advantage of wireless communication is an important subject of network information theory [1].

Most of the previous work focused on one of the following two scenarios:

1) to deliver the same messages to each of the receivers, usually known as the multicast problem; and

2) to deliver completely distinct messages to different receivers, namely the private message problem.

Formally, the distinction between these two broadcast scenarios can be identified by the configurations of the message sets associated with each of the receivers. For the multicast problem, the intended message sets for each of the receivers are identical. For the private message problem, the intended message sets for each of the receivers are mutually exclusive. Clearly, the appropriate coding architecture depends on the configurations of the message sets.

Between these two "extreme" broadcast scenarios, the multicast and the private message problems, there is a rich collection of "intermediate" problems with message sets of interesting configurations and significant engineering appeal. A good example is the degraded message set problems first considered in [2], which can be used to model broadcast scenarios with a progressively encoded source and receivers of different quality-of-service requirement.

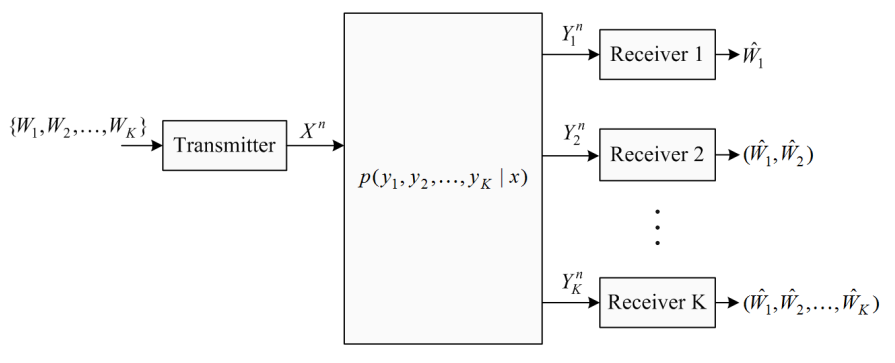

Fig. 1. Broadcast channel with degraded message sets.

Fig. 1 illustrates a general discrete memoryless broadcast channel with degraded message sets. The transmitter has a total of $K$ independent messages $\left(M_{1}, M_{2}, \ldots, M_{K}\right)$. Each of the $K$ receivers demands a subset of messages from the transmitter. The message set $\mathcal{S}_{k}$ intended for receiver $k$ is given by

$$
\mathcal{S}_{k}=\left\{M_{1}, M_{2}, \ldots, M_{k}\right\}, \quad k=1,2, \ldots, K .
$$

Clearly, we have

$$
\mathcal{S}_{1} \subseteq \mathcal{S}_{2} \subseteq \cdots \subseteq \mathcal{S}_{K}
$$

and hence the name "degraded message sets".

For the degraded message set problem, there is a natural communication strategy based on superposition coding [3] and direct decoding. With $K$ independent messages at the transmitter and $K$ receivers, an $K$-layer superposition code can be built with the $k$ th layer from the bottom representing message $M_{k}$. Receiver $k$ decodes messages $\left(W_{1}, W_{2}, \ldots, W_{k}\right)$ by directly decoding all the bottom layers up to the $k$ th. For $K=2$, it was shown in [2] that this natural strategy is also optimal in achieving the capacity region of the channel. For $K \geq 3$, however, finding the capacity region of the discrete memoryless broadcast channel with degraded message sets remains an open problem in network information theory.

In an excellent contribution [4], Nair and El Gamal considered a special three-receiver discrete memoryless broadcast channel with degraded message sets and presented a precise single-letter characterization of the capacity region. Specifically, in [4], it was assumed that:

1) receiver 2 is degraded with respect to receiver 1, i.e., $X-Y_{1}-Y_{2}$ forms a Markov for any input distribution 


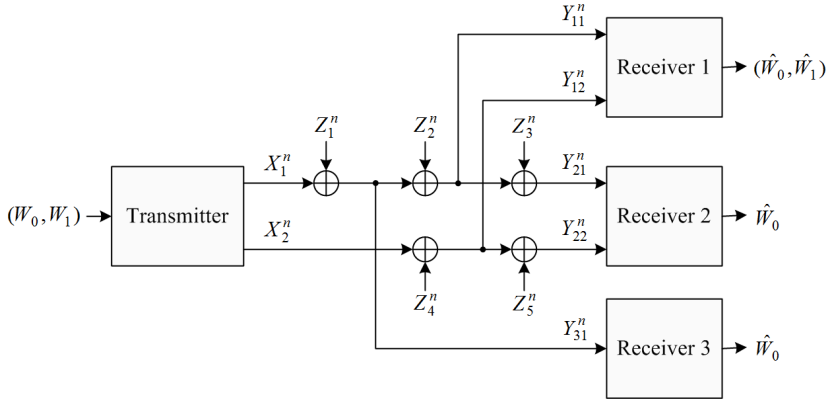

Fig. 2. Product Gaussian broadcast channel with degraded message sets.

$p(x) ;$ and

2) the rate of message $M_{2}$ is set to be zero so in defacto, there are only two independent messages $M_{1}$ and $M_{3}$ at the transmitter.

Under these two assumptions, Nair and El Gamal [4] proved a surprising result that the natural scheme that uses direct decoding is, in general, suboptimal. Instead, a coding scheme that uses indirect decoding [4] can always achieve the capacity region of the channel.

Building on the result of [4], in this paper, we consider a specific product Gaussian broadcast channel with degraded message sets and provide an explicit characterization of the capacity region. The main tools used in this characterization are Lagrangian theory [5] and an extremal entropy inequality of Liu and Viswanath [6]. It is worth mentioning that the exact same product Gaussian model was also considered in the original work of Nair and El Gamal [4], and characterizing the capacity region was posted as an open problem.

\section{Channel Model}

As shown in Fig. 2, consider a discrete-time memoryless product Gaussian broadcast channel with three receivers. At each time sample, the received signals at receivers 1, 2 and 3 are given by $Y_{1}=\left(Y_{11}, Y_{12}\right), Y_{2}=\left(Y_{21}, Y_{22}\right)$ and $Y_{3}=Y_{31}$, respectively, where

$$
\begin{aligned}
& Y_{31}=X_{1}+Z_{1}, \quad Y_{11}=Y_{31}+Z_{2}, \quad Y_{21}=Y_{11}+Z_{3} \\
& Y_{12}=X_{2}+Z_{4}, \quad Y_{22}=Y_{12}+Z_{5} \text {. }
\end{aligned}
$$

Here, $X=\left(X_{1}, X_{2}\right)$ is the channel input, and $Z_{i}, i=$ $1,2,3,4,5$, are Gaussian noise with zero means with covariance $N_{i}$, respectively, and are assumed to be mutually independent of each other. We consider two different types of power constraints on the channel input $X$ : an average total power constraint

$$
E\left[X_{1}^{2}+X_{2}^{2}\right] \leq P
$$

and an individual per-subchannel power constraint

$$
E\left[X_{i}^{2}\right] \leq P_{i}, \quad i=1,2 .
$$

The transmitter has two independent messages $M_{0}$ and $M_{1}$, where $M_{0}$ is a common message intended for all three receivers and $M_{1}$ is a private message intended only for receiver 1 . The capacity region $\mathcal{C}(P)$ is given by the set of nonnegative rate pairs $\left(R_{0}, R_{1}\right)$ that can be achieved by any coding scheme under the average total power constraint (2). Likewise, the capacity region $\mathcal{C}\left(P_{1}, P_{2}\right)$ is given by the set of nonnegative rate pairs $\left(R_{0}, R_{1}\right)$ that can be achieved by any coding scheme under the individual per-subchannel power constraint (3).

From the channel model (1), it is clear that $X-Y_{1}-Y_{2}$ forms a Markov for any distribution on the channel input $X$. In this case, a single-letter characterization of the capacity region was obtained in [4, Prop. 2] and is given by the set of nonnegative rate tuples $\left(R_{0}, R_{1}\right)$ such that

$$
\begin{aligned}
R_{0} & \leq I\left(U_{1} ; Y_{21}\right)+I\left(U_{2} ; Y_{22}\right) \\
R_{0} & \leq I\left(V_{1} ; Y_{31}\right) \\
R_{1} & \leq I\left(X_{1} ; Y_{11} \mid U_{1}\right)+I\left(X_{2} ; Y_{12} \mid U_{2}\right) \\
R_{0}+R_{1} & \leq I\left(V_{1} ; Y_{31}\right)+I\left(X_{1} ; Y_{11} \mid V_{1}\right)+I\left(X_{2} ; Y_{12} \mid U_{2}\right)
\end{aligned}
$$

for some joint distributions on $\left(U_{1}, V_{1}, X_{1}\right)$ and $\left(U_{2}, X_{2}\right)$ such that $U_{1}-V_{1}-X_{1}$ forms a Markov chain. The main goal of this paper is to evaluate the rate region (4) for the specific product Gaussian model (1) under both average total and individual per-subchannel power constraints.

\section{Main Results}

The main result of the paper is an explicit characterization of the capacity region of the product Gaussian broadcast channel (1) under the individual per-subchannel power constraint (3), summarized in the following theorem.

Theorem 1: The capacity region $\mathcal{C}\left(P_{1}, P_{2}\right)$ of the threereceiver product Gaussian broadcast channel (1) under the individual per-subchannel power constraint (3) is given by the set of nonnegative rate tuple $\left(R_{0}, R_{1}\right)$ such that

$$
\begin{aligned}
R_{0} & \leq C\left(\frac{P_{1}-Q_{1}}{Q_{1}+N_{1}+N_{2}+N_{3}}\right)+C\left(\frac{P_{2}-Q_{2}}{Q_{2}+N_{4}+N_{5}}\right) \\
R_{0} & \leq C\left(\frac{P_{1}}{N_{1}}\right) \\
R_{1} & \leq C\left(\frac{Q_{1}}{N_{1}+N_{2}}\right)+C\left(\frac{Q_{2}}{N_{4}}\right) \\
R_{0}+R_{1} & \leq C\left(\frac{P_{1}}{N_{1}}\right)+C\left(\frac{Q_{2}}{N_{4}}\right)
\end{aligned}
$$

for some $0 \leq Q_{1} \leq P_{1}$ and $0 \leq Q_{2} \leq P_{2}$, where $C(x):=$ $\frac{1}{2} \log (1+x)$.

As a corollary, we have the following characterization of the capacity region of the product Gaussian broadcast channel (1) under the average total power constraint (2).

Corollary 1: The capacity region $\mathcal{C}(P)$ of the three-receiver product Gaussian broadcast channel (1) under the average total power constraint (2) is given by the set of nonnegative rate tuple $\left(R_{0}, R_{1}\right)$ such that

$$
\begin{aligned}
R_{0} & \leq C\left(\frac{Q_{3}}{Q_{1}+N_{1}+N_{2}+N_{3}}\right)+C\left(\frac{Q_{4}}{Q_{2}+N_{4}+N_{5}}\right) \\
R_{0} & \leq C\left(\frac{Q_{1}+Q_{3}}{N_{1}}\right) \\
R_{1} & \leq C\left(\frac{Q_{1}}{N_{1}+N_{2}}\right)+C\left(\frac{Q_{2}}{N_{4}}\right) \\
R_{0}+R_{1} & \leq C\left(\frac{Q_{1}+Q_{3}}{N_{1}}\right)+C\left(\frac{Q_{2}}{N_{4}}\right)
\end{aligned}
$$

for some $Q_{i} \geq 0, i=1,2,3,4$, and $Q_{1}+Q_{2}+Q_{3}+Q_{4} \leq P$. 
Proof: This is a simple consequence of Theorem 1 and the well-known fact that

$$
\mathcal{C}(P)=\bigcup_{P_{1}+P_{2} \leq P} \mathcal{C}\left(P_{1}, P_{2}\right) .
$$

The rest of the paper is devoted to the proof of Theorem 1 .

\section{Proof OF THEOREM 1}

The achievability of the rate region (5) follows from that of (4) by setting $X_{i}=U_{i}+W_{i}$ for $i=1,2$ and $V_{1}=X_{1}$, where $U_{i}$ and $W_{i}$ are two independent Gaussian variables with zero means and variances $P_{i}-Q_{i}$ and $Q_{i}$, respectively. (Note that for such a choice of $\left(U_{1}, V_{1}, X_{1}\right), U_{1}-V_{1}-X_{1}$ forms a trivial Markov chain.) We therefore concentrate on proving the converse part of the theorem.

To prove the converse part of the theorem, we shall need the following extremal entropy inequality which first appeared in $[6$, Th. 8].

Lemma 1 ([6]): Let $P$ and $\mu$ be two nonnegative real numbers, and let $Z_{1}, Z_{2}$ be two Gaussian variables with zero means and variances $N_{1}$ and $N_{2}$, respectively. Assume that $0<N_{1} \leq N_{2}$. If there exists a nonnegative real number $P^{*}$ satisfying

$$
\begin{aligned}
\left(P^{*}+N_{1}\right)^{-1}+M_{1} & =\mu\left(P^{*}+N_{2}\right)^{-1}+M_{2} \\
M_{1} P^{*} & =0 \\
M_{2}\left(P-P^{*}\right) & =0
\end{aligned}
$$

for some nonnegative real numbers $M_{1}$ and $M_{2}$, then

$$
\begin{aligned}
h(X & \left.+Z_{1} \mid U\right)-\mu h\left(X+Z_{2} \mid U\right) \\
& \leq \frac{1}{2} \log 2 \pi e\left(P^{*}+N_{1}\right)-\frac{\mu}{2} \log 2 \pi e\left(P^{*}+N_{2}\right)
\end{aligned}
$$

for any $(X, U)$ independent of $\left(Z_{1}, Z_{2}\right)$ and such that $E\left[X^{2}\right] \leq P$.

We are now ready to prove the converse part of the theorem. Consider proof by contradiction. Let $\left(R_{0}^{o}, R_{1}^{o}\right)$ be an achievable rate pair that lies outside the rate region (5). From [7], we have $R_{0}^{o} \leq R_{0}^{\max }$ where

$$
R_{0}^{\max }:=\min \left\{C\left(\frac{P_{1}}{N_{1}+N_{2}+N_{3}}\right)+C\left(\frac{P_{2}}{N_{4}+N_{5}}\right), C\left(\frac{P_{1}}{N_{1}}\right)\right\} .
$$

Note that when $R_{1}^{o}=0, R_{0}^{\max }$ can be achieved by letting $Q_{1}=Q_{2}=0$ in (5). Thus, we may assume that $R_{1}^{o}>0$ and write $R_{1}^{o}=R_{1}^{*}+\delta$ for some $\delta>0$, where $R_{1}^{*}$ is given by

$$
\begin{aligned}
\max & R_{1} \\
\text { s.t. } & R_{0}^{o} \leq C\left(\frac{P_{1}-Q_{1}}{Q_{1}+N_{1}+N_{2}+N_{3}}\right)+C\left(\frac{P_{2}-Q_{2}}{Q_{2}+N_{4}+N_{5}}\right) \\
R_{1} & \leq C\left(\frac{Q_{1}}{N_{1}+N_{2}}\right)+C\left(\frac{Q_{2}}{N_{4}}\right) \\
R_{0}^{o}+R_{1} & \leq C\left(\frac{P_{1}}{N_{1}}\right)+C\left(\frac{Q_{2}}{N_{4}}\right) \\
Q_{1} & \leq P_{1} \\
Q_{2} & \leq P_{2} \\
-Q_{1} & \leq 0 \\
-Q_{2} & \leq 0 .
\end{aligned}
$$

Let $\left(R_{1}^{*}, Q_{1}^{*}, R_{2}^{*}\right)$ be an optimal solution to the above optimization problem. Then, $\left(R_{1}^{*}, Q_{1}^{*}, R_{2}^{*}\right)$ must satisfy the Karush-Kuhn-Tucker (KKT) conditions [5] as shown in the top of next page, where $T_{i}, i=1,2,3,4$, and $M_{i}, i=1,2,3,4$, are nonnegative Lagrangian multipliers. From the KKT conditions (9)-(12), we have

$$
\begin{aligned}
&\left(T_{1}+T_{3}\right) R_{0}^{o}+R_{1}^{o} \\
&=\left(T_{1}+T_{3}\right) R_{0}^{o}+R_{1}^{*}+\delta \\
&=\left(T_{1}+T_{3}\right) R_{0}^{o}+\left(T_{2}+T_{3}\right) R_{1}^{*}+\delta \\
&= T_{1} R_{0}^{o}+T_{2} R_{1}^{*}+T_{3}\left(R_{0}^{o}+R_{1}^{*}\right)+\delta \\
&= T_{1}\left[C\left(\frac{P_{1}-Q_{1}^{*}}{Q_{1}^{*}+N_{1}+N_{2}+N_{3}}\right)+\right. \\
&\left.C\left(\frac{P_{2}-Q_{2}^{*}}{Q_{2}^{*}+N_{4}+N_{5}}\right)\right]+T_{2}\left[C\left(\frac{Q_{1}^{*}}{N_{1}+N_{2}}\right)+\right. \\
&\left.C\left(\frac{Q_{2}^{*}}{N_{4}}\right)\right]+T_{3}\left[C\left(\frac{P_{1}}{N_{1}}\right)+C\left(\frac{Q_{2}^{*}}{N_{4}}\right)\right]+\delta \\
&= T_{1}\left[C\left(\frac{P_{1}-Q_{1}^{*}}{Q_{1}^{*}+N_{1}+N_{2}+N_{3}}\right)+\right. \\
&\left.C\left(\frac{P_{2}-Q_{2}^{*}}{Q_{2}^{*}+N_{4}+N_{5}}\right)\right]+T_{2} C\left(\frac{Q_{1}^{*}}{N_{1}+N_{2}}\right)+ \\
& T_{3} C\left(\frac{P_{1}}{N_{1}}\right)+C\left(\frac{Q_{2}^{*}}{N_{4}}\right)+\delta .
\end{aligned}
$$

On the other hand, by the KKT condition (9) and the assumption that $\left(R_{0}^{o}, R_{1}^{o}\right)$ is achievable, we have

$$
\begin{aligned}
\left(T_{1}+\right. & \left.T_{3}\right) R_{0}^{o}+R_{1}^{o} \\
= & \left(T_{1}+T_{3}\right) R_{0}^{o}+R_{1}^{o} \\
= & \left(T_{1}+T_{3}\right) R_{0}^{o}+\left(T_{2}+T_{3}\right) R_{1}^{o} \\
= & T_{1} R_{0}^{o}+T_{2} R_{1}^{*}+T_{3}\left(R_{0}^{o}+R_{1}^{o}\right) \\
\leq & T_{1}\left[I\left(U_{1} ; Y_{21}\right)+I\left(U_{2} ; Y_{22}\right)\right]+ \\
& T_{2}\left[I\left(X_{1} ; Y_{11} \mid U_{1}\right)+I\left(X_{2} ; Y_{12} \mid U_{2}\right)\right]+ \\
& T_{3}\left[I\left(V_{1} ; Y_{31}\right)+I\left(X_{1} ; Y_{11} \mid V_{1}\right)+I\left(X_{2} ; Y_{12} \mid U_{2}\right)\right] \\
= & T_{1} h\left(Y_{21}\right)+T_{1} h\left(Y_{22}\right)+T_{3} h\left(Y_{31}\right)- \\
& {\left[h\left(Y_{11} \mid X_{1}\right)+h\left(Y_{12} \mid X_{2}\right)\right]+} \\
& {\left[T_{2} h\left(Y_{11} \mid U_{1}\right)-T_{1} h\left(Y_{21} \mid U_{1}\right)\right]+} \\
& {\left[h\left(Y_{12} \mid U_{2}\right)-T_{1} h\left(Y_{22} \mid U_{2}\right)\right]+} \\
& T_{3}\left[h\left(Y_{11} \mid V_{1}\right)-h\left(Y_{31} \mid V_{1}\right)\right]
\end{aligned}
$$

for some joint distributions on $\left(U_{1}, V_{1}, X_{1}\right)$ and $\left(U_{2}, X_{2}\right)$ such that $U_{1}-V_{1}-X_{1}$ forms a Markov chain and $E\left[X_{i}^{2}\right] \leq P_{i}$ for $i=1,2$.

The terms on the right-hand side of the above equation can be further bounded/evaluated as follows.

1) It is well known [1] that Gaussian maximizes differential entropy for a given power, so we have

$$
\begin{aligned}
& h\left(Y_{21}\right) \leq \frac{1}{2} \log 2 \pi e\left(P_{1}+N_{1}+N_{2}+N_{3}\right) \\
& h\left(Y_{22}\right) \leq \frac{1}{2} \log 2 \pi e\left(P_{2}+N_{4}+N_{5}\right) \\
& h\left(Y_{31}\right) \leq \frac{1}{2} \log 2 \pi e\left(P_{1}+N_{1}\right) .
\end{aligned}
$$

2) The channel inputs $\left(X_{1}, X_{2}\right)$ are independent of the 


$$
\begin{aligned}
T_{2}\left(Q_{1}^{*}+N_{1}+N_{2}\right)^{-1}+M_{1} & =T_{1}\left(Q_{1}^{*}+N_{1}+N_{2}+N_{3}\right)^{-1}+M_{2} \\
\left(Q_{2}^{*}+N_{4}\right)^{-1}+M_{3} & =T_{1}\left(Q_{2}^{*}+N_{4}+N_{5}\right)^{-1}+M_{4} \\
T_{2}+T_{3} & =1 \\
T_{1} R_{0}^{o} & =T_{1}\left[C\left(\frac{P_{1}-Q_{1}^{*}}{Q_{1}^{*}+N_{1}+N_{2}+N_{3}}\right)+C\left(\frac{P_{2}-Q_{2}^{*}}{Q_{2}^{*}+N_{4}+N_{5}}\right)\right] \\
T_{2} R_{1}^{*} & =T_{2}\left[C\left(\frac{Q_{1}^{*}}{N_{1}+N_{2}}\right)+C\left(\frac{Q_{2}^{*}}{N_{4}}\right)\right] \\
T_{3}\left(R_{0}^{o}+R_{1}^{*}\right) & =T_{3}\left[C\left(\frac{P_{1}}{N_{1}}\right)+C\left(\frac{Q_{2}^{*}}{N_{4}}\right)\right] \\
M_{1} Q_{1}^{*} & =0 \\
M_{2}\left(P_{1}-Q_{1}^{*}\right) & =0 \\
M_{3} Q_{2}^{*} & =0 \\
M_{4}\left(P_{2}-Q_{2}^{*}\right) & =0
\end{aligned}
$$

Gaussian noise $\left(Z_{1}, Z_{2}, Z_{3}, Z_{4}, Z_{5}\right)$, so we have

$$
\begin{gathered}
h\left(Y_{11} \mid X_{1}\right)=h\left(Z_{1}+Z_{2}\right)=\frac{1}{2} \log 2 \pi e\left(N_{1}+N_{2}\right) \\
h\left(Y_{12} \mid X_{1}\right)=h\left(Z_{4}\right)=\frac{1}{2} \log 2 \pi e N_{4} .
\end{gathered}
$$

3) Putting together the KKT conditions (7), (13) and (14), we have

$$
\begin{aligned}
T_{2}\left(Q_{1}^{*}+N_{1}+N_{2}\right)^{-1}+M_{1}= & T_{1}\left(Q_{1}^{*}+N_{1}+\right. \\
& \left.N_{2}+N_{3}\right)^{-1}+M_{2} \\
M_{1} Q_{1}^{*}= & 0 \\
M_{2}\left(P_{1}-Q_{1}^{*}\right)= & 0
\end{aligned}
$$

where $M_{1}, M_{2}, T_{1}$ and $T_{2}$ are nonnegative real numbers. By Lemma $1^{1}$, we have

$$
\begin{aligned}
& T_{2} h\left(Y_{11} \mid U_{1}\right)-T_{1} h\left(Y_{21} \mid U_{1}\right) \\
&= T_{2} h\left(X_{1}+Z_{1}+Z_{2} \mid U_{1}\right)- \\
& T_{1} h\left(X_{1}+Z_{1}+Z_{2}+Z_{3} \mid U_{1}\right) \\
& \leq \frac{T_{2}}{2} \log 2 \pi e\left(Q_{1}^{*}+N_{1}+N_{2}\right)- \\
& \frac{T_{1}}{2} \log 2 \pi e\left(Q_{1}^{*}+N_{1}+N_{2}+N_{3}\right) .
\end{aligned}
$$

4) Similarly, putting together the KKT conditions (8), (15) and (16), we have

$$
\begin{aligned}
\left(Q_{2}^{*}+N_{4}\right)^{-1}+M_{3} & =T_{1}\left(Q_{2}^{*}+N_{4}+N_{5}\right)^{-1}+M_{4} \\
M_{3} Q_{2}^{*} & =0 \\
M_{4}\left(P_{2}-Q_{2}^{*}\right) & =0
\end{aligned}
$$

where $M_{3}, M_{4}$ and $T_{1}$ are nonnegative real numbers.

${ }^{1}$ If $T_{2}=0$, we have either $T_{1}=0$ or $Q_{1}^{*}=0$. In either case, inequality (21) holds trivially.
Again, by Lemma 1, we have

$$
\begin{aligned}
& h\left(Y_{12} \mid U_{2}\right)-T_{1} h\left(Y_{22} \mid U_{2}\right) \\
&= h\left(X_{2}+Z_{4} \mid U_{2}\right)-T_{1} h\left(X_{2}+Z_{4}+Z_{5} \mid U_{2}\right) \\
& \leq \frac{1}{2} \log 2 \pi e\left(Q_{2}^{*}+N_{4}\right)- \\
& \frac{T_{1}}{2} \log 2 \pi e\left(Q_{2}^{*}+N_{4}+N_{5}\right) .
\end{aligned}
$$

5) Finally, note that

$$
\begin{aligned}
h\left(Y_{11} \mid V_{1}\right)-h\left(Y_{31} \mid V_{1}\right) \\
\quad=h\left(X_{1}+Z_{1}+Z_{2} \mid V_{1}\right)-h\left(X_{1}+Z_{1} \mid V_{1}\right) \\
\quad=I\left(Z_{2} ; X_{1}+Z_{1}+Z_{2} \mid V_{1}\right) \\
\quad=h\left(Z_{2}\right)-h\left(Z_{2} \mid X_{1}+Z_{1}+Z_{2}, V_{1}\right) \\
\quad \leq h\left(Z_{2}\right)-h\left(Z_{2} \mid X_{1}+Z_{1}+Z_{2}, V_{1}, X_{1}\right) \\
\quad=h\left(Z_{2}\right)-h\left(Z_{2} \mid Z_{1}+Z_{2}, V_{1}, X_{1}\right) \\
\quad=h\left(Z_{2}\right)-h\left(Z_{2} \mid Z_{1}+Z_{2}\right) \\
\quad=I\left(Z_{2} ; Z_{1}+Z_{2}\right) \\
\quad=\frac{1}{2} \log 2 \pi e\left(N_{1}+N_{2}\right)-\frac{1}{2} \log 2 \pi e N_{1}
\end{aligned}
$$

where (23) is due to the independence of $Z_{2}$ and $V_{1}$; (24) is due to the fact that conditioning reduces differential entropy [1]; and (25) is due to the independence of $\left(Z_{1}, Z_{2}\right)$ and $\left(V_{1}, X_{1}\right)$.

Substitute (19)-(22) and (26) into (18). With some rearranging of terms, we may obtain

$$
\begin{aligned}
\left(T_{1}+T_{3}\right) R_{0}^{o}+R_{1}^{o} \\
\leq T_{1}\left[C\left(\frac{P_{1}-Q_{1}^{*}}{Q_{1}^{*}+N_{1}+N_{2}+N_{3}}\right)+\right. \\
\left.\quad C\left(\frac{P_{2}-Q_{2}^{*}}{Q_{2}^{*}+N_{4}+N_{5}}\right)\right]+T_{2} C\left(\frac{Q_{1}^{*}}{N_{1}+N_{2}}\right)+ \\
T_{3} C\left(\frac{P_{1}}{N_{1}}\right)+C\left(\frac{Q_{2}^{*}}{N_{4}}\right)
\end{aligned}
$$

Note that $\delta>0$, so this is a contradiction to (17). We therefore 
conclude that any achievable rate pair $\left(R_{0}^{o}, R_{1}^{o}\right)$ must also be inside the rate region (5). This completes the proof of the converse part of the theorem.

\section{ACKNOWLEDGMENT}

The work of J. W. Yoo and T. Liu was supported by the National Science Foundation under Grant CCF-08-45848 and CCF-09-16867. The work of Y. Liang was supported by the National Science Foundation under Grant CCF-09-15772.

\section{REFERENCES}

[1] T. M. Cover and J. A. Thomas, Elements of Information Theory. New York: Wiley, 1991.

[2] J. Körner and K. Marton, "General broadcast channels with degraded message sets," IEEE Trans. Inf. Theory, vol. IT-23, no. 1, pp. 60-64, Jan. 1977.

[3] P. P. Bergmans, "Random coding theorem for broadcast channels with degraded components," IEEE Trans. Inf. Theory, vol. IT-19, no. 2, pp. 197-207, Mar. 1973.

[4] C. Nair and A. El Gammal, "The capacity region of a class of threereceiver broadcast channels with degraded message sets," IEEE Trans. Inf. Theory, vol. 55, no. 10, pp. 4479-4493, Oct. 2009.

[5] S. Boyd and L. Vandenberghe, Convex Optimization. Cambridge University Press, 2004.

[6] T. Liu and P. Viswanath, "An extremal inequality motivated by multiterminal information-theoretic problems," IEEE Trans. Inf. Theory, vol. 53, no. 5 , pp. 1839-1851, May 2007.

[7] D. Blackwell, L. Breiman, and A. Thomasian, "The capacity of a class of channels," Ann. Math. Statist., vol. 30, no. 4, pp. 1229-1241, Dec. 1959. 